\section{Lymphocytic hypophysitis pre- senting as a visual threatening sellar mass and its complete recovery with oral steroids: think beyond surgery}

\author{
Pramila Dharmshaktu, Mahaveer Singh, \\ Dinesh Dhanwal \\ Department of Medicine, Endocrinology \\ Division, Maulana Azad Medical College, \\ New Delhi, India
}

\section{Abstract}

A 37-years female patient presented to us with hypothyroidism. She was started on oral thyroid replacement and sent back to home. Four weeks later she again came with history of progressive vision loss and headache. Ophthalmological evaluation revealed bitemporal hemianopia with bilateral papilloedema. Non-contrast computed tomography revealed mass lesion in pituitary fossa. Hormone profile showed secondary hypocortisolism and secondary hypothyroidism. Magnetic resonance imaging (MRI) brain showed diffusely enlarged sellar mass with suprasellar extension with enhancement of both anterior, posterior pituitary andinfundibulam. Neurologist advised urgent surgical intervention. A diagnosis of lymphocytic hypophysitis was made based on clinical and radiological evidences and surgery was deferred. Patient was given oral steroid on OPD basis and she improved dramatically. The mass also vanished subsequently on follow up MRI brain. This case illustrates the potential benefit of trial of medical management when there is a strong possibility even with vision loss due to mass effect in lymphocytic hypophysitis.

\section{Introduction}

Lymphocytic hypophysitis is a rare clinical condition characterized by inflammation of pituitary gland followed by fibrosis of the gland. Pituitary insufficiency develops after some time. It commonly appears as a pituitary mass with homogeneous enhancement after gadolinium injection and a thickened infundibulum, making it difficult to distinguish from a pituitary tumor. There is no definite biochemical, serological markers or radiological imaging for this disorder and the diagnosis can only be clearly established by histological examination. The anterior pituitary is characteristically infiltrated by polyclonal $\mathrm{T}$ and B lymphocytes together with plasma cells. Its treatment is aimed at reducing the size of the enlarged pituitary. Lympholytic drugs such as glucocorticoids, azathioprine, and methotrexate, pituitary surgery and radiotherapy are the current approaches. Here we are presenting a case in which patient responded dramatically to steroid, establishing diagnosis of the lymphocytic hypophysitis.

\section{Case Report}

A 37-years female patient from army background presented to us with complaints of fatigue, loss of energy and weight gain since one month. Examination revealed cold and dry skin. A TSH and Free T4 were ordered for the patient. The results were as follows TSH: 7.41 uIU/mL (normal range: 1.49-5.5 uIU/mL), FT4: $0.98 \mathrm{ng} / \mathrm{mL}$ (normal range: $0.8-1.61 \mathrm{ng} / \mathrm{mL}$ ). TSH was slightly increased. Anti TPO antibody was also positive with higher values. Correlating with the history, physical examination and laboratory investigation, a diagnosis of hypothyroidism was made. Patient was started on oral levothyroxine 50 ug daily. Patient was sent back to home with an advice of follow up. She improved with thyroid hormone replacement. Few weeks later patient returned back to our hospital with headache and vision loss of two week duration. Headache was chronic progressive, diffuse, used to increase on lying down position and worst in the morning. Her ophthalmological evaluation showed bilateral papilledema and bitemporal visual field defect. Complete blood count revealed $\mathrm{Hb}$ of $10.8 \%$ gm, TLC of 7900 cells/cumm, platelet count of $1.8 \mathrm{lac} /$ cumm and ESR of $49 \mathrm{~mm}$ in 1st hour. Kidney function tests and liver function tests were within normal limit. Chest X-ray was also normal. Pituitary hormone profile revealed serum prolactin of $14.2 \mathrm{ng} / \mathrm{mL}$, serum LH OF $1.91 \mathrm{mu} / \mathrm{mL}$, serum FSH of $1.52 \mathrm{mU} / \mathrm{mL}$, serum cortisol of 0.49 $\mathrm{ug} / \mathrm{dL}$ and basal growth hormone of $0.70 \mathrm{ng} / \mathrm{mL}$. Suspecting intracranial space occupying lesion, non contrast CT scan was ordered for her which showed sellar mass. Neurology consultation was taken and they advised an urgent surgical intervention to be required for her. MRI brain with gadolinium contrast was subsequently planned which revealed a $1.5 \times 2.3 \times 1.3$ diffuse sellar mass with supra sellar extension causing compression of the optic chiasma and uniform sellar floor depression (Figure 1). The mass shows a rapid homogeneous delayed (>90 sec) triangular enhancement after gadolinium with involvement of the adjacent dura (dural tail). A diagnosis of lymphocytic hypophysitis was made based on clinical and radiological evidences and surgery was deferred. We planned for noninvasive
Correspondence: Pramila Dharmshaktu, A-404, Crown Apartments, Sector 7, Dwarka, Plot 18-B, New Delhi, India. Tel. +91.958.293.4837

E-mail: dr.pramila09@gmail.com

Key words: lymphocytic hypophysistis, hypothyroidism, steroids.

Acknowledgements: I would like to thank my patient for her cooperation and wish for her good health.

Contributions: PD and MS are the treating residents and mainly responsible for drafting and in conception of the case report; DD has given the ultimate guidance regarding the management of the patient and guided us in making of this case report.

Conflict of interests: the authors declare no potential conflict of interests.

Received for publication: 2 September 2013. Revision received: 14 September 2013.

Accepted for publication: 15 September 2013

This work is licensed under a Creative Commons Attribution NonCommercial 3.0 License (CC BYNC 3.0)

(C) Copyright P. Dharmshaktu et al., 2013 Licensee PAGEPress, Italy

Endocrinology Studies 2013; 3:e9

doi:10.4081/es.2013.e9

approach. She was started on oral tablet prednisolone at a dose of $40 \mathrm{mg}$ daily with close monitoring of her visual field. With treatment she started noticing improvement in her vision and complaints. Visual field assessment after 2 week showed improvement. She was no more complaining of headache.

After 6 weeks again a detailed clinical, ophthalmological and radiological evaluation was done. The sellar mass also vanished subsequently on follow up MRI brain (Figures 2 and $3)$. Patient is now on regular follow up.

This case illustrates the potential benefit of trial of medical management when there is strong possibility of lymphocytic hypophysitis. Steroid can prevent the morbidity of the surgical intervention in a this case and can support the diagnosis as well.

\section{Results and Discussion}

Autoimmune hypophysitis is a rare inflammatory condition of the pituitary gland. Lymphocytic autoimmune hypophysitis, the more common form was first described in 1962 by Goudie and Pinkerton. ${ }^{1}$ It causes enlargement of the pituitary gland. The clinical presentations with headaches, visual field abnor- 
malities and more rarely diplopia are due to extrasellar pituitary enlargement with displacement and compression of optic chiasma. Hypopituitarism is caused by inflammatory destruction of the pituitary and compression of the residual normal gland. ACTH deficiency is usually the earliest and most common hormonal impairment and rarely can induce an acute onset secondary adrenal insufficiency. ${ }^{2-4}$

Lymphocytic hypophysitis seems to be strongly correlated with pregnancy. It frequently affects women in last six months of pregnancy and in the first six months after delivery. ${ }^{5}$ Symptoms of seller compression as headache and visual disturbances are the most common and usually the initial presenting complaints. Headache is found in $60 \%$ of patients and considered due to distension and distortion of the dura mater by the expanding pituitary mass. ${ }^{3}$ The pituitary volume does not correlate well with severity, duration, and frequency of headache, suggesting that the headache associated with pituitary masses may not be simply a structural problem. Visual abnormalities is found in $43 \%$ of patients and include visual field defects and decreased acuity that are secondary to compression of the optic chiasm by the upwardly expanding pituitary mass. Next most common are symptoms due to a partial or complete deficit of the anterior pituitary hormones, mainly ACTH (43\%) followed by TSH (18\%), gonadotropins (12\%), and PRL (11\%). These defects are considered the direct result of the autoimmune distruction of the pituitary acinar cells. They produce the classic signs and symptoms of hypoadrenalism, hypothyroidism, and hypogonadism. The deficit of PRL manifests itself in the postpartum as inability to lactate.

The gold standard for the diagnosis of lymphocytic hypophysitis is pituitary biopsy and its histopathological examination. ${ }^{5}$ Hormonal evaluation can also add in diagnosis. On histopathological examination, the adenohypophysis is infiltrated by lymphoplasmacytic cells consisting lymphocytes, plasma cells and macrophages. T lymphocytes are significantly more abundant than B lymphocytes. Lymphoplasmacytic aggregates surround atrophic acini of pituitary cells, with remaining areas of reactive fibrosis. ${ }^{6}$

The NMR characteristics are uniform seller floor depression and an extrasellar pituitary enlargement displacing the optic chiasma. The mass shows a characteristic homogeneous delayed (>90 sec) triangular enhancement after gadolinium contrast with involvement of adjacent dura. ${ }^{7}$

The immunofluorescence method is one of the most widely used current methods to detect pituitary autoantibodies. ${ }^{8}$ ACTH deficiency indicates that the initial auto antigens targeted by the immune system reside within the corticotrophs. ${ }^{9}$

The treatment of lymphocytic hypophysitis is only symptomatic which includes reduction of the pituitary mass with replacement of the deficient hormone. Mass reduction can be achieved by pituitary surgery, lympholytic drugslike glucocorticoids, azathioprine, or radiotherapy. Surgery had been the most common form of treatment in the past. Surgery is usually performed via the endoscopic transphenoidal approach, which needs only minimal invasive surgery.

Glucocorticoids can be effective for treating it both as an antiinflammatory agents to reduce the size and as replacement of defective adrenal function. The most commonly used glucocorticoids have been prednisone at a dose of 20 to $60 \mathrm{mg}$ per day. Diagnosis of lym-
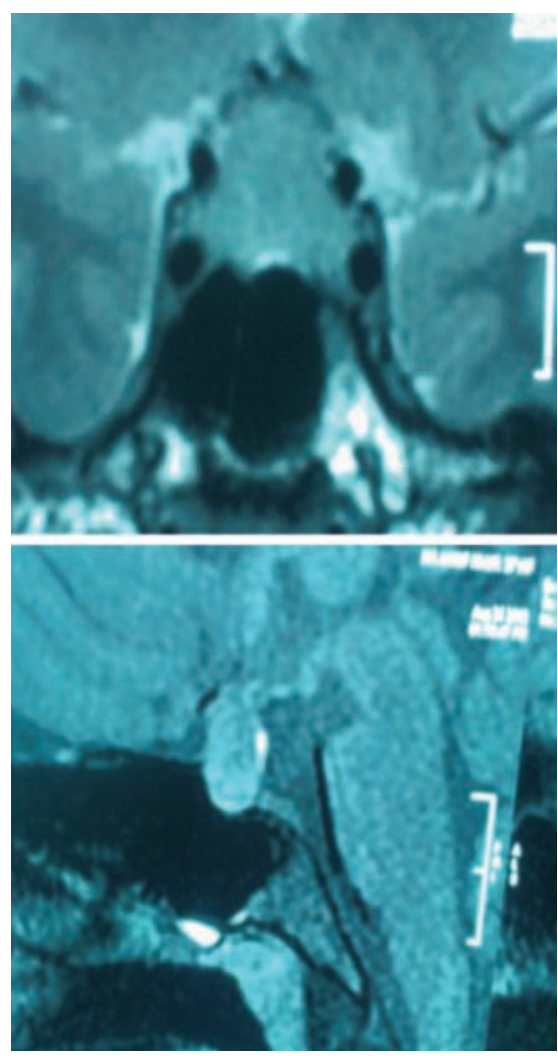

Figure 1. Magnetic resonance imaging without contrast showing coronal and sagittal section of brain showing sellar mass with parasellar extension
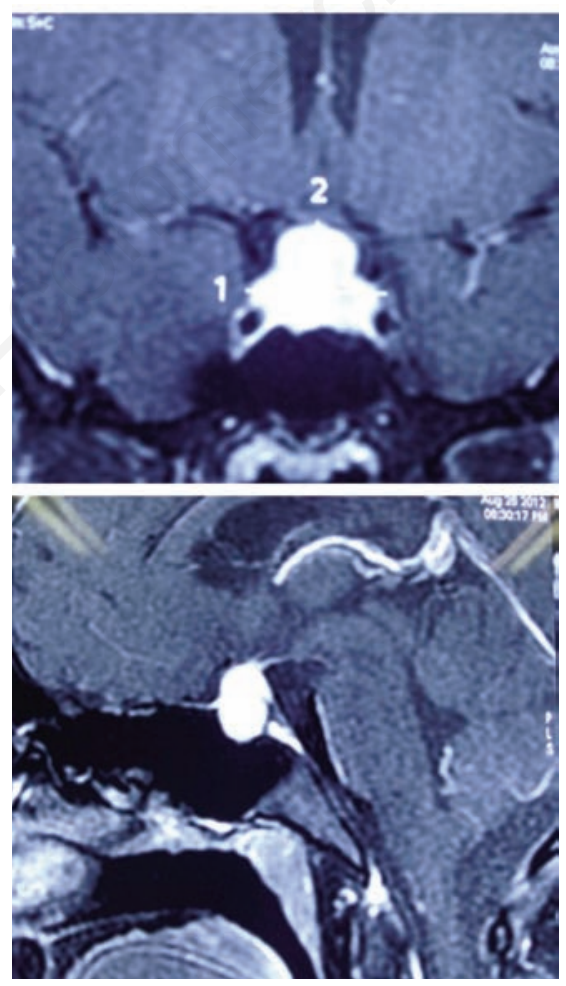

Figure 2. Post contrast magnetic resonance imaging of the brain in coronal and sagittal section showing well enhancing sellar mass with enhancement of infundibulam and parietal loss of posterior pituitary bright spot.
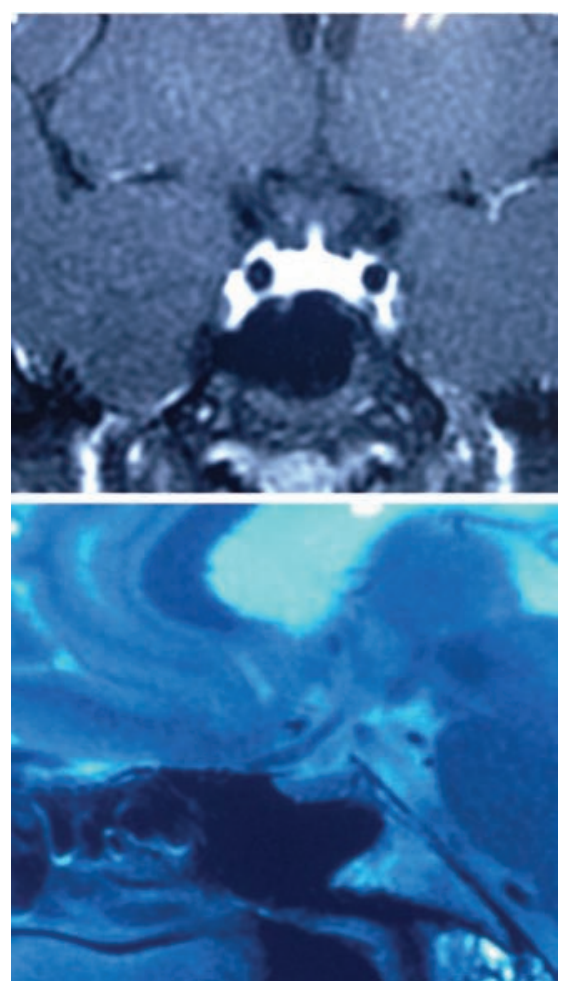

Figure 3. Post contrast magnetic resonance imaging of the brain following steroid therapy shows complete disappearance of pituitary mass. 
phocytic hypophysitis can be confirmed upon spontaneous remission on corticosteroid treatment in patients with suspected autoimmune hypophysitis. In the absence of a serum marker, rapid response to treatment as a diagnostic marker avoids more invasive diagnostic procedures.

\section{Conclusions}

Our case was successfully treated by oral steroids only and was prevented from intracranial surgery. So here we have a case where patient responded dramatically to steroid, establishing diagnosis of the lymphocytic hypophysitis.

\section{References}

1. Ito K, Mukawa J, Miyagi $\mathrm{K}$, et al. Lymphocytic adenohypophysitis with sudden onset of diabetes insipidus in menopausal female. Neurol Med Chir (Tokyo) 1992;32:346-50.

2. Bellastella A, Bizzarro A, Coronella C, et al. Lymphocytic hypophysitis: a rare or underestimated disease? Eur J Endocrinol 2003;149:363-76.

3. Rivera JA. Lymphocytic hypophysitis: disease spectrum and approach to diagnosis and therapy. Pituitary 2006;9:35-45.

4. Mirakian R, Cudworth AG, Bottazzo GF, et al. Autoimmunity to anterior pituitary cells and the pathogenesis of insulin-dependent diabetes mellitus. Lancet 1982;1:755-9.

5. De Bellis A, Ruocco G, Battaglia M, et al. Immunological and clinical aspects of lym- phocytic hypophysitis. Clin Sci (Lond) 2008;114:413-21.

6. Abstracts of the 54th Annual Meeting of the German Society of Neuropathology and Neuroanatomy (DGNN): Neuropathology in the 21st Century, Düsseldorf, Germany, September 16 - 19, 2009. Acta Neuropathol 2009;118:433-67.

7. Ünlühizarci K, Bayram F, Çolak R, et al. Distinct radiological and clinical appearance of lymphocytic hypophysitis. J Clin Endocrinol Metabol 2001;86:1861-4.

8. Gutenberg A, Buslei R, Fahlbusch R, et al. Immunopathology of primary hypophysitis: implications for pathogenesis. Am J Surg Pathol 2005;29:329-38.

9. Escobar-Morreale H, Serrano-Gotarredona J, Varela C. Isolated adrenocorticotropic hormone deficiency due to probable lymphocytic hypophysitis in a man. J Endocrinol Investig 1994;17:127. 